\title{
Increased cerebral responses to salient transitions between alternating stimuli in chronic migraine with medication overuse headache and during migraine attacks
}

2019, Vol. 39(8) 988-999

(C) International Headache Society 2019 Article reuse guidelines: sagepub.com/journals-permissions DOI: I0.II77/03331024|8825359 journals.sagepub.com/home/cep

(S)SAGE

\author{
Volodymyr B Bogdanov ${ }^{1,2} \odot$, Olena V Bogdanova ${ }^{1,2}$, \\ Alessandro Viganò $\dot{2}^{2,3,4}$, Quentin Noirhomme ${ }^{5,6}$, \\ Steven Laureys ${ }^{5,6}$, Radhouane Dallel ${ }^{7,8,9}$, \\ Christophe Phillips ${ }^{5,10} \odot$ and Jean Schoenen ${ }^{2}$
}

\begin{abstract}
Introduction: In a previous study exploring central pain modulation with heterotopic stimuli in healthy volunteers, we found that transitions between sustained noxious and innocuous thermal stimulations on the foot activated the "salience matrix". Knowing that central sensory processing is abnormal in migraine, we searched in the present study for possible abnormalities of these salient transitional responses in different forms of migraine and at different time points of the migraine cycle.

Methods: Participants of both sexes, mostly females, took part in a conditioned pain modulation experiment: Migraineurs between $(n=14)$ and during attacks $(n=5)$, chronic migraine patients with medication overuse headache $(n=7)$ and healthy volunteers $(n=24)$. To evoke the salience response, continuous noxious cold or innocuous warm stimulations were alternatively applied on the right foot. Cerebral blood oxygenation level dependent responses were recorded with fMRI.

Results: Switching between the two stimulations caused a significant transition response in the "salience matrix" in all subject groups (effect of the condition). Moreover, some group effects appeared on subsequent post-hoc analyses. Augmented transitional blood oxygenation level dependent responses in the motor cortex and superior temporal sulcus were found in two patient groups compared to healthy controls: chronic migraine with medication overuse headache patients and migraineurs recorded during an attack. In chronic migraine with medication overuse headache patients, salience-related responses were moreover greater in the premotor cortex, supplementary motor area, lingual gyrus and dorso-medial prefrontal cortex and other "salience matrix" areas, such as the anterior cingulate and primary somatosensory cortices.

Conclusion: This study shows salience-related hyperactivation of affective and motor control areas in chronic migraine with medication overuse headache patients and, to a lesser extent, in episodic migraine patients during an attack. The greater extension of exaggerated blood oxygenation level dependent responses to unspecific salient stimuli in chronic migraine with medication overuse headache than during a migraine attack could be relevant for headache chronification.
\end{abstract}

\section{Keywords}

Episodic migraine, chronic migraine, medication overuse headache, salience matrix, fMRI, BOLD responses, stimulus transition

Date received: 30 April 20I8; revised: 14 November 2018; accepted: 23 November 2018

'Centre de Recherche en Neurosciences de Lyon Inserm, UI 028 - CNRS UMR5292 Bâtiment Inserm, Bron, France

${ }^{2}$ Headache Research Unit, Department of Neurology, CHR Citadelle, University of Liège, Liège, Belgium

${ }^{3}$ Department of Human Neurosciences, Sapienza, University of Rome, Rome, Italy

${ }^{4}$ Department of Neurology and Psychiatry, La Sapienza, University of Rome, Rome, Italy

${ }^{5}$ GIGA institute, University of Liège, Liège, Belgium

${ }^{6}$ Coma Science Group, Department of Neurology, University and

University Hospital of Liège, Liège, Belgium
${ }^{7}$ Université Clermont Auvergne, Clermont-Ferrand, France

${ }^{8} \mathrm{CHU}$ Clermont-Ferrand, Clermont-Ferrand, France

${ }^{9}$ Inserm, Neuro-Dol, Clermont-Ferrand, France

${ }^{10}$ Department of Electrical Engineering and Computer Science, University of Liège, Liège, Belgium

Corresponding author:

Volodymyr B Bogdanov, Centre de Recherche en Neurosciences de Lyon, Inserm U1028 - CNRS UMR5292 Bâtiment Inserm, 16 avenue Doyen Lépine, 69676 Bron, France. Email: vlabogd@yahoo.com 


\section{Abbreviations}

ACC: anterior cingulate cortex; BOLD signal: blood oxygenation level dependent signal; S1: primary somatosensory cortex; S2: secondary somatosensory cortex; SMA: supplementary motor cortex; STS: superior temporal sulcus; HV: healthy volunteers; MnA: interictal episodic migraine patients; MA: episodic migraineurs during an attack; $\mathrm{CM}-\mathrm{MOH}$ : chronic migraine with medication overuse headache.

\section{Background}

While episodic migraine is characterized by recurrent attacks separated by symptom-free intervals, its complication, chronic migraine $(\mathrm{CM})$, produces quasi daily headache and is most frequently favoured by overuse of acute migraine drugs causing so-called medication overuse headache (MOH) (1).

Headache chronification may involve an impairment of central pain control mechanisms. Cortical processing of painful stimuli is altered in CM patients (2) who also have hyper-excitability of sensory cortices to innocuous stimuli (3). The cerebral network previously termed the "pain matrix" (4) includes the anterior cingulate cortex, insula, SI, SII, medial temporal cortex and prefrontal cortex. In fact, besides being activated by noxious stimuli, these areas also respond to highly salient innocuous stimulations (5), hence the rewording in "salience matrix".

Migraine patients have increased connectivity of "salience matrix" regions (anterior insula and anterior and mid-cingulate cortex) with other brain areas (6). It has been suggested that enhanced engagement of the salience network in migraine patients results in impaired ability to disengage attention from ongoing pain (7). Brain activations by noxious and innocuous stimuli between and during migraine attacks were found to be abnormal in a number of studies comprehensively reviewed by Schwedt et al. (8). In migraine patients compared to healthy controls, interictal pain-induced activations were greater in the dorsolateral prefrontal cortex, temporal pole, postcentral gyrus, anterior and middle cingulate cortex, hippocampus, parahippocampal gyrus and lentiform nuclei, but smaller in the precentral gyrus, secondary somatosensory cortex and superior temporal gyrus. Stimulus-induced activation is more pronounced during than outside of an attack in the insular cortex, temporal pole, occipital cortex, parahippocampal gyrus, posterior thalamus or amygdala, depending on sensory modality.

In $\mathrm{MOH}$, functioning of various areas belonging to the "salience matrix" including the insula, posterior parietal lobe, orbitofrontal cortex and thalamus was altered (9). Mechanical pain-induced fMRI BOLD activation was reduced in several areas of the lateral pain system such as the parietal cortex and supramarginal gyrus (10). Morphometric MRI studies showed decreased gray matter volume (11) and reduced thickness in the orbitofrontal cortex, but higher local gyrifications in temporal and occipital cortices (12). Functional connectivity at rest in $\mathrm{MOH}$ patients was decreased between the precuneus and regions of the default-mode network (frontal and parietal cortices), but increased between the precuneus and hippocampal/temporal areas (13).

We have previously shown in healthy volunteers that switching between noxious and innocuous sustained thermal stimuli induces a widespread nonspecific activation of the "salience matrix" areas (14). In the present study, we investigated with fMRI whether this salience response is abnormal in migraine patients between and during attacks, and in $\mathrm{MOH}$ patients.

\section{Materials and methods}

\section{Subjects and experimental protocol}

The healthy volunteers and migraine patients aged 18 65 years were recruited from the personnel of Centre Hospitalier Régional de la Citadelle, Liège, Belgium, and students of the University of Liège, Belgium. The project was registered with EudraCT and the Belgian Federal Agency for Medicines and Health Products (B70720084343) and was approved by the Ethics Committee of the University Hospital (Liège, Belgium) (ref 2008/160). Twenty-four healthy male and female volunteers (mean age: 31.3 years, range: 24-45 years) took part in the experiment (HV group). None of the healthy subjects had a chronic pain, neurological or psychiatric disorder.

A structured interview allowed identification of migraine patients according to the criteria of ICHDII: Codes 1.1, 1.3 and 8.2 (1). The criteria of inclusion comprised age, the ability to speak French, not being on antidepressant (tricyclic or SSRI) treatment and not taking prophylactic antimigraine treatment. Subjects with cognitive impairments or major handicap, cerebral hypertension or previous cerebral trauma, language difficulties, alcoholism, cancer or contraindication for an MRI scan were not included in the study. After recruitment for the study, $\mathrm{MOH}$ patients diagnosed according to ICHD-II criteria filled in a paper headache diary for at least 1 month to document headache and medication overuse before undergoing the recordings. We recorded 14 episodic migraine without aura patients (four males, mean age: 32.8 years, range $18-47$ years) outside of an attack (interictal: $\geq 3$ days after and before an attack) (MnA group), five patients during an attack (ictal, one male, mean age 33.6 years, range 20-58 years) (MA 
group) and seven female patients suffering from chronic migraine with medication overuse headache $(\mathrm{CM}-\mathrm{MOH}$, mean age 44.4 years, range $24-60$ years). The ongoing headache intensity during fMRI recordings was estimated using a visual analogue scale from 0 to 10 .

All subjects signed an informed consent form. They were only aware of the general purpose of our experiment; that is, study of the impact of cold on pain and physiological responses induced by laser heat stimuli. They were thus naïve about our working hypothesis. Healthy subjects received a financial compensation for their willingness to participate in the study and the time spent in the laboratory. Patients were paid for their travel expenses.

The experimental protocol was the same as in our previous study (14). We aimed to test the effect of heterotopic noxious conditioning on phasic test pain responses; that is, a central pain modulation mechanism that is impaired in chronic pain. Laser heat stimuli on the left hand were combined with continuous noxious cold alternating with innocuous lukewarm stimulations applied using compresses on the right foot. In the present study we focused on the cerebral responses to the heterotopic conditioning stimulation. There were 10 blocks of noxious cold (120 ms each) and 10 blocks of innocuous warm stimulation $(100 \mathrm{~ms}$ each). The pain levels were controlled as in our previous study (14). Analysis of pain level data did not reveal any relation with other results, and they will not be discussed herein.

The Cogent 2000 software delivered auditory stimuli to the operator who stayed all the time in the scanning room next to the subject, informing him when to manually replace the compresses (Cogent 2000 developed by the Cogent 2000 team at the FIL and the ICN and Cogent Graphics developed by John Romaya at the LON at the Wellcome Department of Imaging Neuroscience).

\section{Structural and functional MRI data acquisition}

MRI data were acquired on a 3T head-only scanner (Magnetom Allegra, Siemens Medical Solutions, Erlangen, Germany) using a standard transmit-receive quadrature head coil.

A structural MRI was acquired in a separate session 20-30 minutes before the functional acquisition. For the structural scan, a high-resolution T1-weighted image was obtained for each subject (3D MDEFT (15); $\mathrm{TR}=7.92 \mathrm{~ms}, \quad \mathrm{TE}=2.4 \mathrm{~ms}, \quad \mathrm{TI}=910 \mathrm{~ms}, \quad \mathrm{FA}=15^{\circ}$, $\mathrm{FoV}=256 \times 256 \times 176 \mathrm{~mm}^{3}, \quad 1 \mathrm{~mm}$ isotropic spatial resolution, 176 sagittal slices, $1 \mathrm{~mm}$ thickness, acquisition time: 12 minutes).

Multislice $\mathrm{T} 2 *$-weighted functional images were acquired with a gradient-echo echo-planar imaging sequence using an axial slice orientation and covering the whole brain/most of the brain ( 34 slices, $\mathrm{FoV}=$ $192 \times 192 \mathrm{~mm}^{2}$, voxel size $3 \times 3 \times 3 \mathrm{~mm}^{3}, \quad 25 \%$ interslice gap, matrix size $64 \times 64 \times 34$, TR $=2040 \mathrm{~ms}$, $\mathrm{TE}=30 \mathrm{~ms}, \mathrm{FA}=90^{\circ}$ ). The three initial volumes were discarded to avoid $\mathrm{T} 1$ saturation effects.

Field map data were also acquired to unwarp the functional images: Acquisition time: TR/TE: 517/4.92 and $7.38 \mathrm{~ms}$, Resolution: $64 \times 64$, Slices: 32 slices $(3 \mathrm{~mm}$ thickness, $30 \%$ gap), Voxel size: $3.4 \times 3.4 \times 3 \mathrm{~mm}^{3} ; \mathrm{FoV}$ : $220 \times 220 \mathrm{~mm}^{2}, F A: 90^{\circ}$, Bandwidth: $260 \mathrm{~Hz} / \mathrm{Px}$.

\section{sMRI and fMRI data analysis}

The fMRI images were processed using the "Statistical Parametric Mapping" software (SPM8; Wellcome Trust Centre for Neuroimaging, University College London, $\mathrm{UK}$; http//www.fil.ion.ucl.ac.uk/spm) implemented in MATLAB (Mathworks Inc., Sherborn, MA).

Functional image time series were corrected for motion and distortion using the "Realign and Unwarp" tool (16) together with the Fieldmap Toolbox (17). The high-resolution T1 image was then co-registered with the functional images and segmented into grey matter, white matter, and cerebrospinal fluid (18). Functional images were spatially normalized to the Montreal Neurological Institute (MNI) space (voxel size: $3 \times 3 \times 3 \mathrm{~mm}^{2}$ ) using the normalization parameters obtained from the segmentation procedure and, subsequently, smoothed with a Gaussian kernel with full-width at half-maximum of $6 \mathrm{~mm}$.

Statistical analysis was performed using a general linear model and summary statistics approach. At the first level, data were subjected to high-pass filtering using a cut-off period of 280 seconds. Each boxcar or impulse stimulus function was convolved with a canonical hemodynamic response function as implemented in SPM8. Explicit masks were created from the brain mask image available in SPM8. The binary mask was created by thresholding the brain mask image at a level of 0.5 intensity units. This mask fitted the average normalized anatomical image of our subject group well.

The first $10 \mathrm{~s}$ after switching from warm to cold or from cold to warm were modelled as "early" response. This was used in other studies (19) and allowed good differentiation between early and continuous phase responses. After model estimation, specific effects were tested with appropriate linear contrasts of the parameter estimates, resulting in a contrast value for each voxel. We computed the contrast for early nonspecific responses to transitions between warm and cold applications on the foot.

The contrast images were included in a second-level group analysis using a random-effects approach, treating inter-subject variability as a random factor. 
First, at the second level, we performed an omnibus F-test for detection of overall transition response within four groups.

Then we used an F-test with a contrast for one-way ANOVA to estimate any group effects. We used an undirected search, looking for responses with $p<0.05$ family wise error rate (FWER) corrected for the whole brain volume both at the voxel and cluster levels. Voxel-level significant responses were $p<0.05$ FWER corrected, while cluster level significance was computed under $p<0.001$ uncorrected cluster-forming threshold.

The direction of the group effect was verified by specific post-hoc t-tests with contrasts for the difference between $\mathrm{HV}$ and patient groups where the major difference of the contrast estimates was observed.

Since the greatest post-hoc group effects were found in $\mathrm{CM}-\mathrm{MOH}$ versus $\mathrm{HV}$ contrast, we verified if the areas of maximal overall transition response (S1, insula, thalamus and ACC) showed differential activation along with areas yielded by undirected search. In order to verify if there was an effect with minimal correction, we applied inclusive masks of respective cerebral areas to the t-contrast $\mathrm{HV}<\mathrm{CM}-\mathrm{MOH}$ with cluster forming threshold $p<0.001$, and estimated both peak and cluster significance of the effect.

\section{Head displacement analysis}

Head displacements can reflect motor reactions induced by the compress replacement during the scanning procedure and were computed at realignment of functional scans obtained during the whole scanning session. Time series of the sagittal motion parameter estimated by SPM at realignment of functional images were $1000 \mathrm{~Hz}$ interpolated and partitioned in $10 \mathrm{sec}$ bins locked accurately to the transition time. Within each bin, peak-to peak displacements was calculated. We segregated displacements occurring during the first 10 seconds after changing from cold to warm compress or vice versa and those occurring during the following 90 seconds of sustained cold or warm stimulation. Withinsubject average displacements were compared between four groups of subjects and the significance of group effect was calculated with the Multiple Comparisons (2-tailed) Kruskal-Wallis test.

\section{Results}

Switching between noxious cold and innocuous warm sustained foot stimulation or vice versa induced a short-lasting but widespread BOLD response in all groups of subjects that was maximal in the somatosensory cortex (Figure 1). The activation by the stimulation switch was a non-specific salience response, and it did not differ between the transition from noxious cold to innocuous lukewarm and the inverse transition (Figure 1). Besides S1, the posterior insula/S2, thalamus and anterior cingulate cortex were other areas activated by the transition. The "salience" response in brain regions belonging to the "salience matrix" was significant in all subject groups $(p<0.05 \mathrm{FWE})$ (Figure 2).

However, in MA and CM-MOH patients, this activation was significantly greater in many other brain areas, mostly on the left side of the brain (Table 1, Figure 3). The effect did not differ between responses to onset or offset of cold (Figure 3).

There were a number of frontal, occipital and temporal areas where a significant group effect was found (Table 1, Figure 4). Post-hoc test confirmed that in

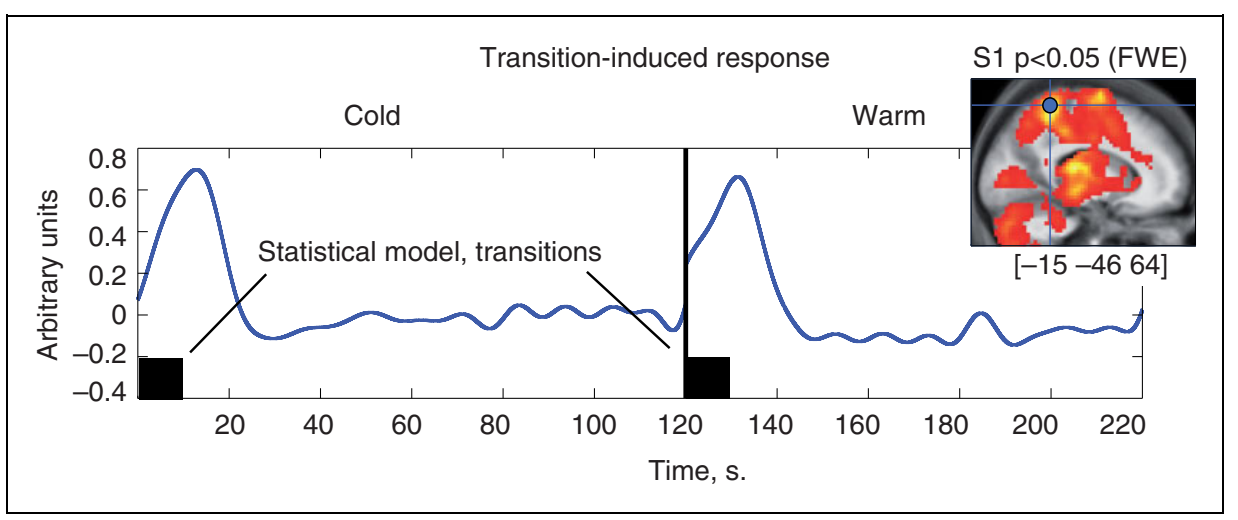

Figure I. Average hemodynamic response in somatosensory cortex SI (shown in the right upper corner) to warm/cold and cold/ warm transitions in all subjects $(n=50)$. The BOLD signal was extracted from a $6 \mathrm{~mm}$ radius spherical VOI at $[-15-4664] \mathrm{MNI}(\mathrm{blue}$ circle of statistical parametric map in insert). Eigenvariates were normalized to the median value of an individual time series, epoched and individually averaged for cold (left) and lukewarm (right) conditions and the group average was estimated (spline interpolation $1000 \mathrm{~Hz}$, low pass filtering $10 \mathrm{~Hz}$ ). 


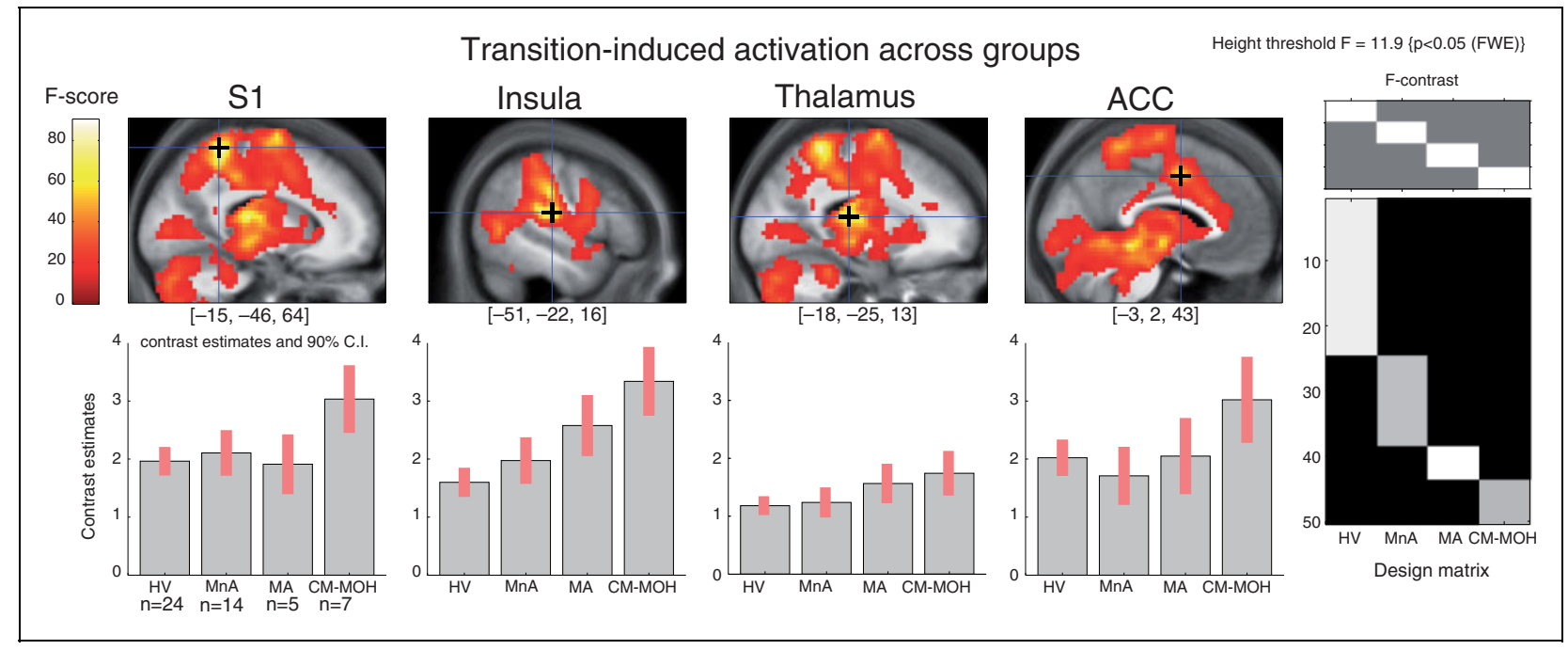

Figure 2. Noxious-innocuous transition-induced activation of salience matrix areas in healthy volunteers (HV), migraine patients between attacks (MnA) and during an attack (MA), and in patients suffering from chronic migraine with medication overuse headache (CM-MOH). Upper: statistical parametric maps overlaid on a sagittal section of averaged individual normalized structural TI images; crosshairs indicate peak activations in SI, insula, thalamus and anterior cingulate cortex. Lower: contrast estimates $( \pm 90 \% \mathrm{Cl})$ of the local statistical peak. $x, y$ and $z$ coordinates according to $\mathrm{MNI}$ space.

Table I. Cerebral areas with a group effect in cerebral activation during transition between warm and cold or vice versa. ANOVA results are shown on the left and post-hoc t-tests for respective areas on the right. Different patterns of group effect are indicated by colors: Red $(\mathrm{HV}, \mathrm{MnA})<(\mathrm{MA}, \mathrm{CM}-\mathrm{MOH})$, yellow $(\mathrm{HV}, \mathrm{MnA}, \mathrm{MA})<\mathrm{CM}-\mathrm{MOH}$ and green $\mathrm{HV}<\mathrm{MnA}<\mathrm{MA}<\mathrm{CM}-\mathrm{MOH}$. Post-hoc peaks belonging to the same STS cluster are labeled respectively with index ${ }^{a}{ }^{*} p<0.05$ whole brain FWER corrected, either voxel or cluster level, depending on whether coordinates or cluster size value, $\mathrm{k}_{E}$ is labeled.

\begin{tabular}{|c|c|c|c|c|c|c|}
\hline \multicolumn{3}{|l|}{ ANOVA } & \multicolumn{4}{|l|}{ Post-hoc t-test } \\
\hline & $\begin{array}{l}\text { MNI peak } \\
\text { coordinates }\end{array}$ & $k_{E}$ & $\begin{array}{l}\text { MA }>\mathrm{HV} \\
\text { contrast } \\
\text { FWER } p<0.05\end{array}$ & $k_{E}$ & $\begin{array}{l}\mathrm{CM}-\mathrm{MOH}>\mathrm{HV} \\
\text { contrast } \\
\text { FWER } p<0.05\end{array}$ & $k_{E}$ \\
\hline \multicolumn{7}{|l|}{$\begin{array}{l}\text { Threshold } \\
\text { FWER } p<0.05\end{array}$} \\
\hline I. Temporo-occipital (STS) & {$\left[\begin{array}{lll}-5 \mid & -55 & \mid 3\end{array}\right]^{*}$} & $\mathrm{II}$ & {$\left[\begin{array}{llll}-51 & -49 & 10\end{array}\right]^{*}$} & 1 & {$\left[\begin{array}{lll}-5 \mid & -55 & 13\end{array}\right]^{*}$} & $36^{\mathrm{a}}$ \\
\hline 2. Temporo-occipital (STS) & {$\left[\begin{array}{lll}-42 & -49 & 13\end{array}\right]^{*}$} & 3 & & & {$\left[\begin{array}{lll}-42 & -49 & 13\end{array}\right]^{*}$} & $36^{\mathrm{a}}$ \\
\hline 3. MI/premotor & {$\left[\begin{array}{lll}-36 & -4 & 46\end{array}\right]^{*}$} & 2 & {$\left[\begin{array}{lll}-36 & -4 & 46\end{array}\right]^{*}$} & 3 & & \\
\hline 4. Premotor & {$\left[\begin{array}{llll}-39 & 5 & 3 & 1\end{array}\right]^{*}$} & 5 & & & {$\left[\begin{array}{llll}-39 & 5 & 3 & 1\end{array}\right]^{*}$} & 48 \\
\hline 5. Premotor & {$\left[\begin{array}{lll}48 & 5 & 19\end{array}\right]^{*}$} & $\mathrm{I}$ & & & {$\left[\begin{array}{lll}48 & 5 & 19\end{array}\right]^{*}$} & 23 \\
\hline 6. SMA/dorsal ACC & {$\left[\begin{array}{lll}9 & 26 & 49\end{array}\right]^{*}$} & 6 & & & {$\left[\begin{array}{lll}9 & 26 & 49\end{array}\right]^{*}$} & 42 \\
\hline \multicolumn{7}{|l|}{$\begin{array}{l}\text { Threshold } \\
\qquad p<0.001 \text { uncorrected }\end{array}$} \\
\hline Temporo-occipital (STS) & {$\left[\begin{array}{lll}48 & -52 & 10\end{array}\right]$} & $249 *$ & & & & \\
\hline Lingual gyrus & {$\left[\begin{array}{lll}-21 & -58 & -2\end{array}\right]$} & $378^{*}$ & & & {$\left[\begin{array}{lll}-24 & -58 & -2\end{array}\right]^{*}$} & 20 \\
\hline Dorso-medial PFC & {$\left[\begin{array}{lll}-12 & 41 & 31\end{array}\right]$} & $159 *$ & & & {$\left[\begin{array}{llll}-1 & 4 & 4 & 3\end{array}\right]^{*}$} & 7 \\
\hline Middle occipital gyrus & {$\left[\begin{array}{lll}30 & -76 & 7\end{array}\right]$} & $140 *$ & & & & \\
\hline Anterior STS & {$[45-1-20]$} & $67^{*}$ & & & & \\
\hline Putamen & {$\left[\begin{array}{lll}33 & -4 & -8\end{array}\right]$} & $86 *$ & & & & \\
\hline
\end{tabular}




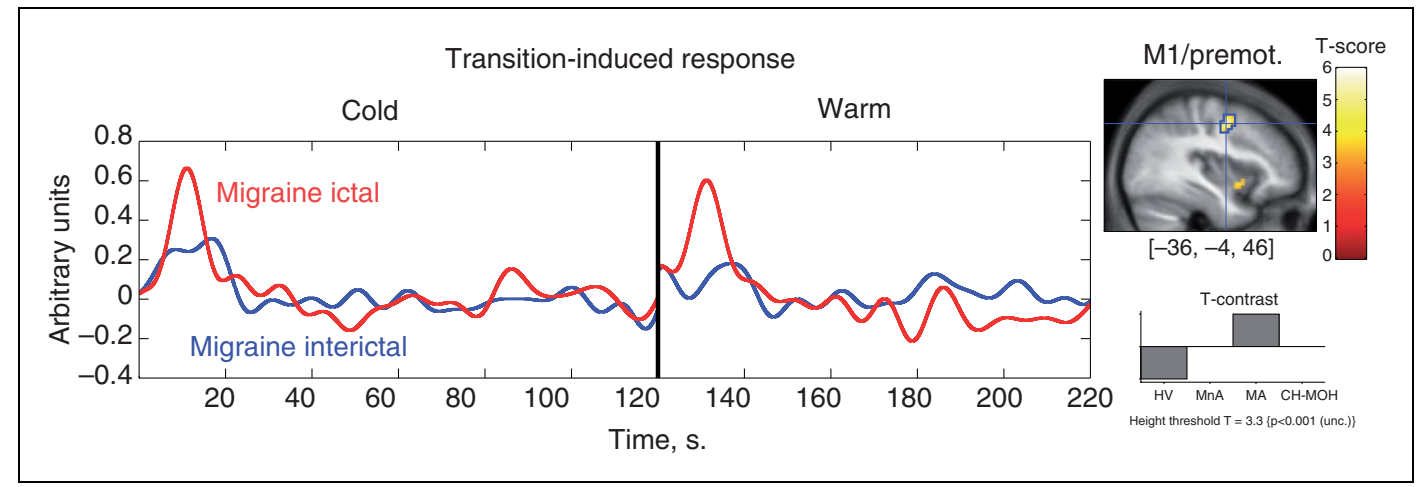

Figure 3. BOLD response to cold/warm transitions in the motor cortex (VOI shown in the left) in migraine patients interictally $(n=14$, blue line) and during an attack $(n=5$, red line). The BOLD signal was extracted from a 48 voxel cluster identified with a $t$-test $(\mathrm{HV}<\mathrm{MA})$ highlighted with blue contour on statistical map on the right inset (signal processed as in Figure I).

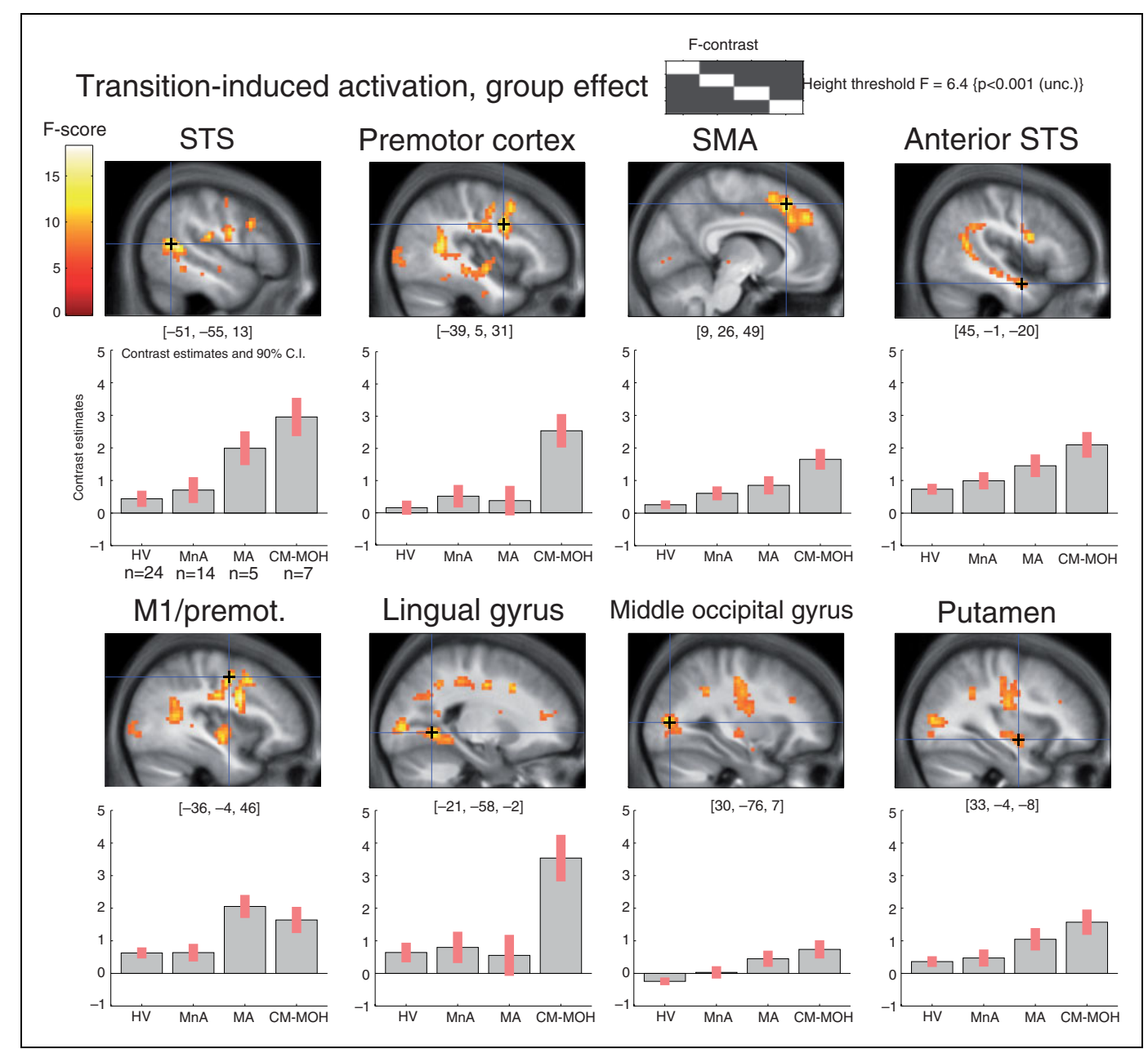

Figure 4. Regional transition-induced activation BOLD responses in the four subject groups. Both migraine patients during an attack (MA) and chronic migraine patients with medication overuse headache $(\mathrm{CM}-\mathrm{MOH})$ have increased responses in the posterior superior temporal sulcus (STS) and MI. In the premotor cortex and lingual gyrus the increased response is only found in the $\mathrm{CM}-\mathrm{MOH}$ group, while in the SMA, anterior STS, middle occipital gyrus and putamen there is a progressive increment of the BOLD response along the sequence $\mathrm{HV}<\mathrm{MnA}<\mathrm{MA}<\mathrm{CM}-\mathrm{MOH}$. For the images, $p<0.00$ I uncorrected threshold has been used. 
migraine patients during an attack (MA), the transition response was greater in the left motor cortex and left posterior superior temporal sulcus compared to healthy volunteers (Table 1, Figure 4). By contrast, there was no significant difference in transitional BOLD activations between interictal migraine patients $(\mathrm{MnA})$ and healthy controls.

In CM-MOH patients, the transitional salience response was also greater than in healthy volunteers in the motor cortex and in the superior temporal sulcus, like in ictal migraine. In addition, in CM$\mathrm{MOH}$ patients there was an increase in activity of the left and right premotor cortex, supplementary motor area, anterior cingulate cortex (ACC), left lingual gyrus and dorso-medial prefrontal cortex (Table 1, Figure 4). ANOVA also revealed increased activation

Table 2. Inclusive masking of the t-test $\mathrm{HV}<\mathrm{CM}-\mathrm{MOH}$ based on the omnibus ANOVA peaks. $p<0.00$ I cluster-forming threshold used. $* p<0.05$ whole brain FWER corrected, either voxel or cluster level, depending on whether coordinates or cluster size value $k_{E}$ is labeled.

\begin{tabular}{lll}
\hline Area/mask & MNI peak coordinates & $k_{E}$ \\
\hline SI & {$\left[\begin{array}{lll}18 & -34 & 67\end{array}\right]$} & $123^{*}$ \\
& {$\left[\begin{array}{lll}-5 I & -7 & 22\end{array}\right]$} & $368^{*}$ \\
Insula & {$\left[\begin{array}{lll}-30 & 17 & -5\end{array}\right]^{*}$} & $160^{*}$ \\
& {$\left[\begin{array}{lll}-36 & -10 & -8\end{array}\right]^{*}$} & \\
Thalamus & {$\left[\begin{array}{lll}18 & -28 & I\end{array}\right]$} & $15 I^{*}$ \\
ACC & {$\left[\begin{array}{lll}-6 & 35 & 22\end{array}\right]$} & $200^{*}$ \\
\hline
\end{tabular}

in the middle occipital gyrus, anterior superior temporal sulcus and putamen.

The hyperactivation of $\mathrm{S} 1$, insula, thalamus and ACC by switching between cold/warm stimulation was confirmed in $\mathrm{CM}-\mathrm{MOH}$ patients at the cluster level by inclusive anatomical masking (Table 2, Figure 5).

We found no correlation between fMRI BOLD data and headache intensity during the recordings in MA or $\mathrm{CM}-\mathrm{MOH}$ subjects.

To search for differences in head movements during the experimental procedure, the head motion parameters were extracted from the realignment parameters estimated by the SPM software. The between-group effect was significant during the 90 -second period of sustained cold or warm application, but not during the early response. CM-MOH patients disclosed greater head displacements than healthy volunteers. We found no significant difference in head motion between migraine patients during an attack and healthy volunteers at any time point (Figure 6).

\section{Discussion}

We found significant early non-specific "salience" BOLD responses at the onset and offset of noxious cold or innocuous lukewarm stimulation (cold/warm transition) in healthy subjects, which is concordant with our previous findings (14), but also in migraine patients during and outside of an attack, and in patients suffering from chronic migraine with medication overuse headache $(\mathrm{CM}-\mathrm{MOH})$. These responses

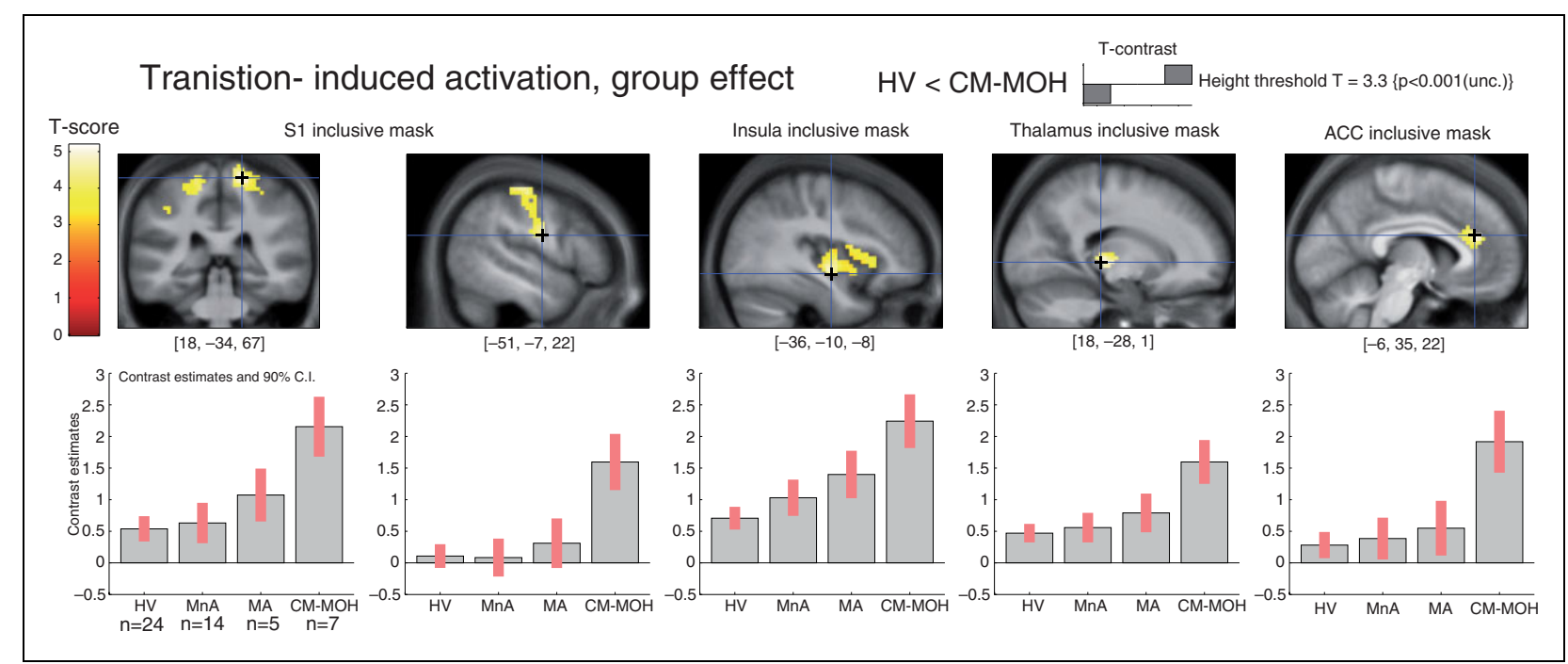

Figure 5. Increased transition-induced activation responses in chronic migraine patients with medication overuse patients (CM$\mathrm{MOH}$ ) in masked "salience matrix" areas. For the images, $p<0.001$ uncorrected cluster forming threshold has been used. See detailed description in the Methods section. 


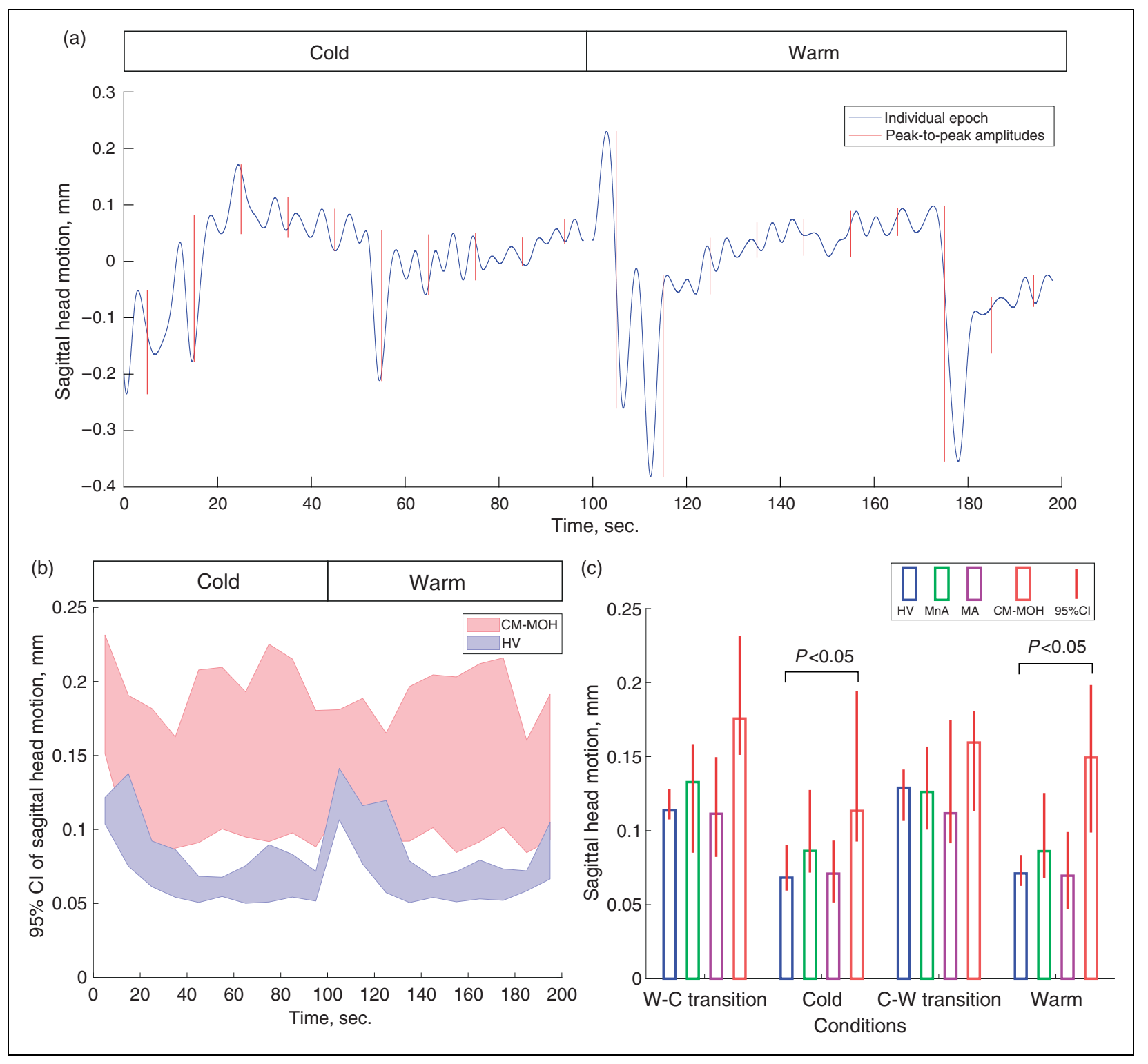

Figure 6. Motion in the scanner in $\mathrm{mm}$ estimated from the sagittal head displacement. (a) Individual example of head motion recorded in the scanner over 200 seconds and peak-to-peak displacements during 10 -second bins. (b) $95 \%$ confidence interval (Cl) of average peak-to-peak head displacements during cold and warm stimulation. $\mathrm{Cl}$ is computed by median bootstrap with $10^{4}$ iterations. (c) Median values of peak-to-peak displacement during 10 -second transitions and 90 -second sustained stimulations. Groups: HV, healthy volunteers $(n=24)$; MnA: migraine patients between attacks $(n=14)$; MA: migraine patients during an attack $(n=5)$, and chronic migraine patients with medication overuse headache (CM-MOH) $(n=7)$.

$*_{p}<0.05$ by Multiple Comparisons (2-tailed) Kruskal-Wallis test. $95 \%$ is computed as in (b).

were observed in a number of cortical and subcortical areas belonging to the "salience matrix". The main novel finding was that during migraine attacks, and even more so in $\mathrm{CM}-\mathrm{MOH}$ patients, the transitional salient activation was significantly augmented in certain areas, while this was not the case in migraine patients between attacks. We will discuss the possible pathophysiological significance of these findings in interictal and ictal migraine, and thereafter in $\mathrm{CM}-\mathrm{MOH}$.
There is clear evidence of altered noxious processing in migraine $(8,20)$; however, in our study, the transitional responses differ only for ictal migraine patients. This could be related to the fact that the transition between noxious and innocuous stimuli in our paradigm represents short-lasting sensory information of a quite different nature compared to that generated by the stimuli used in pain-activation and evoked potential studies. The transitional information, despite 
being salient, may not be sufficiently potent to reveal an increased responsivity of the "salience matrix" in migraine between attacks.

Both ictal migraineurs (MA) and CM-MOH patients had increased unspecific transitional BOLD responses in the motor cortex and superior temporal sulcus. Increased connectivity within several brain networks including motor cortex were observed in migraine patients during an attack (21). Besides that, in magnetoencephalography studies, abnormal cortical excitability is found in migraine patients during the attack (22). These data are in line with the over-reactivity of the motor cortex in our study.

The superior temporal sulcus is a multimodal convergent brain area responsible for multisensory integration due to its bidirectional connections with auditory, visual, and somatosensory cortices and multisensory neurons. It is also known to be activated by affective pain aspects during the watching of painful scenes (23). Though this area was shown to be hypoactivated in migraineurs between attacks (8), its activation during an attack or in chronic migraine may explain both the multimodal hypersensitivity that accompanies the migraine attack rendering patients intolerant to various sensory inputs (24) and the affective symptoms (25).

Unlike migraineurs during an attack, $\mathrm{CM}-\mathrm{MOH}$ patients also displayed overactivation to cold/warm switches in the left lingual gyrus, dorso-medial premotor cortex and supplementary motor area (SMA) and thalamus (Figure 4).

The lingual gyrus is a part of the occipital cortex with a significant role in vision and memory. In migraine patients, this region may be involved in the generation and propagation of the migraine aura (26). Light stimulation-induced activation was stronger in the secondary visual cortex, corresponding to the lingual gyrus region, in migraine patients, which mirrors the effect of concomitant pain and light stimulation in controls (27). Increased lingual gyrus activation in $\mathrm{CM}-\mathrm{MOH}$ patients in our study may be a reflection of increased visual cortex sensitization in the distressing context of chronic migraine headache.

The supplementary motor area and adjacent motor/ premotor cortices, involved in memorizing the dynamic aspects of repeated pain, are overactivated in migraine patients in response to painful stimuli (28). This is in line with our findings in $\mathrm{CM}-\mathrm{MOH}$ patients. A possible cause of the augmented SMA activity in $\mathrm{CM}-\mathrm{MOH}$ patients may also be an increased demand of motor control. During the scanning sessions, all subjects were requested to avoid movements. The analysis of head movements during the neuroimaging recordings showed that $\mathrm{CM}-\mathrm{MOH}$ patients moved significantly more than the other subjects. We have reported the data on head movements in the scanner that is usually considered of no interest to confirm that the difference between $\mathrm{CM}-\mathrm{MOH}$ and the other groups cannot be attributed to motion artefacts. There are several reasons why the different salience-related brain activity between groups is unlikely to be due to head motion. First of all, the motion parameters are included in the fMRI analysis as covariates of no interest, so their impact is not considered in the BOLD response. With regards to the whole brain map, the localization of motion-induced activity is mostly at the edges between the tissues: the surface of the brain and white matterventricle transitions. Greater activity in $\mathrm{CM}-\mathrm{MOH}$ and MA groups is, on the contrary, located mostly in the cortical grey matter; that is, not in locations typical for motion-related artefacts. As shown in Figure 6, head motion is numerically greater in $\mathrm{CM}-\mathrm{MOH}$ patients than in the other groups during all conditions, but this difference is significant during sustained cold and warm applications and not during transitions. It can therefore not explain the specific BOLD changes that only occur at the cold-warm and warm-cold transitions.

The hyperactivation of the SMA that plays a key role in motor control, particularly in the control of internally generated movements and postural body stabilization, may reflect increased mobilisation of brain resources to maintain the same position for a long time. Increased necessity of self-control in $\mathrm{CM}-\mathrm{MOH}$ patients during the cold/warm switch may be also reflected by the augmented BOLD responses in the prefrontal cortex. It is known that the superior frontal gyrus is involved in introspection (29). Specific activation in this area in $\mathrm{CM}-\mathrm{MOH}$ supports our hypothesis that SMA/premotor activation can be due to increased self-focusing due to the experimental paradigm. Greater activation of the motor cortex in $\mathrm{CM}-\mathrm{MOH}$ may be related to the role of the motor cortex in descending antinociceptive controls, which are known to be compromised in this patient cohort and could be in line with the well-documented analgesic effect of motor cortex stimulation in chronic pain syndromes (30).

At first sight, cerebral nonspecific overactivation in $\mathrm{CM}-\mathrm{MOH}$ may seem surprising given that most metabolic and morphometric studies have shown respectively hypometabolism and a decrease of grey matter density or cortical thickness in such patients (9-11,31). Nonetheless, several evoked potential studies in $\mathrm{CM}-\mathrm{MOH}$ patients indicate hypersensitivity of somatosensory cortices to innocuous (32) or noxious stimuli (33). With migraine chronification, the sensory cortices become truly hyperexcitable, which contrasts with their relative hypoexcitability to single stimuli, but hyperresponsivity to repeated stimuli, in episodic migraine between attacks $(32,34)$. Whether this could be related to functional changes in the thalamus where increased tissue volume, including of sensory 
nuclei, was reported in $\mathrm{CM}-\mathrm{MOH}$ patients $(35,36)$ remains to be determined. The electrophysiological profile in chronic migraine thus resembles that of the migraine attack (37), but switches to the interictal pattern when chronic migraine reverses to episodic migraine after adequate treatment (38). A morphological correlate of cortical hypersensitivity in $\mathrm{CM}-\mathrm{MOH}$ could be the recent finding of higher local gyrifications in temporal and occipital cortices (12).

To test further the cerebral overactivation in $\mathrm{CM}-\mathrm{MOH}$ patients, and since the greatest post-hoc group effects were found between the $\mathrm{CM}-\mathrm{MOH}$ and HV groups, we applied an inclusive mask analysis (Figure 5). This approach demonstrated in $\mathrm{CM}-\mathrm{MOH}$ patients an overactivation of the ACC, thalamus, insula and $\mathrm{S} 1$; that is, brain areas belonging to the "salience matrix", previously called the "pain matrix" $(4,5)$.

Activity in these areas correlates with unpleasantness, intensity and affective components of pain perception and depends on other factors like attention, mood and actual situation. This network is also activated by non-painful stimuli as long as they are highly salient. Our results corroborate this concept, since the highly salient switching from warm to cold or from cold to warm induced widespread responses in areas of the "salience matrix" irrespective of the direction of the switch.

Our findings suggest that overall salience reactivity is enhanced during migraine attacks, and even more so in chronic migraine with medication overuse headache. Enhanced engagement of the salience network (7) and increased connectivity of two of its core regions, the anterior insula and anterior cingulate cortex, with other brain areas has already been reported in migraine patients (6), and increased grey matter density was found in the anterior cingulate cortex for the "in pain" phase compared with the interictal period (39).

Hyperreactivity in salience-related brain regions in ictal migraine, and to an even higher degree in CMMOH patients, may reflect a general hypersensitivity to external stimuli or enhanced expectation towards salient events. Whether this hypersensitivity promotes the headache and correlates with allodynia or is facilitated by the pain remains to be determined.

Although we cannot rule it out formally, the possibility that the increased "salience responses" in $\mathrm{CM}-\mathrm{MOH}$ patients could be consequent to an enhanced transmission of thermonociceptive input at spinal level is unlikely for various reasons. First, the fact that we found no correlation between pain perception and fMRI data in this study does a priori not favour a bottom-up mechanism related to increased activity in nociceptive pathways at the spinal or subcortical level. Concordantly, in a study of trigeminal nociception in $\mathrm{CM}-\mathrm{MOH}$ patients, responses to noxious stimuli were chiefly increased centrally and not at the brain stem level (33). Second, in $\mathrm{CM}-\mathrm{MOH}$ we found no significant increase in BOLD responses during sustained cold application or instantaneous noxious laser stimulation. It is nonetheless noteworthy that in our study CM-MOH patients, but not the other subjects, reported pain from application of lukewarm compresses, which suggests that they were allodynic even at an extracephalic site.

Headache intensity during the experimental session, on the other hand, did not impact the fMRI results in $\mathrm{CM}-\mathrm{MOH}$ patients (data not shown), suggesting that the large saliency-related overactivation in multiple brain areas found here is not related to ongoing head pain. Admittedly, the VAS used to assess headache intensity during the recordings may not be reliable or sensitive enough to detect weak correlations. In our study there was an incremental activity change between groups along the sequence (healthy volunteers $<$ interictal migraine $<$ ictal migraine $<\mathrm{CM}-\mathrm{MOH}$ ) in the SMA, middle occipital gyrus, anterior STS, putamen and insula. It remains to be determined whether this might be related to headache frequency and/or chronification.

Our study has some limitations. The low number of patients in the MA and CM-MOH groups is one of them. It is notoriously difficult to recruit migraine patients for imaging studies during spontaneous attacks and during the chronic disabling phase of their disease. We think, however, that these limitations are outweighed by the fact that we were able to record cerebral responses of migraine patients during the attack and in the chronification phase with medication overuse and to compare them with those of patients in the interictal phase and healthy volunteers. The comparison revealed highly significant differences between groups. In spite of our groups not being equilibrated with respect to age, and the $\mathrm{CM}-\mathrm{MOH}$ patients being significantly older than the controls, our supplementary analysis revealed that there is no trend in covariation between age and the activation of the areas of interest in the controls, therefore we suggest that age uniquely cannot explain salience-related hyperactivity in $\mathrm{MOH}$.

To conclude, BOLD responses to unspecific salient stimuli are increased in multisensory brain areas belonging to the "salience matrix" and in certain motor areas during migraine attacks and in a broader network of affective and cognitive cerebral areas in chronic migraine patients with medication overuse. This suggests that increased responsivity to salient sensory stimuli may play a crucial role in migraine headache and its chronification, and headache-associated sensoriphobia as well as possibly allodynia. 


\section{Article highlights}

- Transitions between noxious and innocuous stimuli activate the "salience matrix" areas of the brain.

- BOLD responses to such transitions were recorded in patients with migraine at headache-free and attack phases and in chronic migraine with medication overuse headache.

- Over-activations of affective, motor control and "salience matrix" areas are observed in the two last patient groups.

- The greater BOLD responses to unspecific salient stimuli could be relevant for headache chronification.

\section{Acknowledgements}

We are grateful to Drs André Luxen, Eric Salmon and Pierre Maquet for organizational and to Christian Degueldre for technical assistance.

\section{Declaration of conflicting interests}

The authors declared no potential conflicts of interest with respect to the research, authorship, and/or publication of this article.

\section{Funding}

The authors disclosed receipt of the following financial support for the research, authorship, and/or publication of this article: This study was supported by research convention 3.4.650.09 from the National Fund for Scientific Research, Belgium, to JS, and by a research grant from the CHU of Clermont-Ferrand, France, to RD and JS.

\section{ORCID iD}

Volodymyr B Bogdanov (D) http://orcid.org/0000-0002-71071426

Christophe Phillips (D) http://orcid.org/0000-0002-4990-425X

\section{References}

1. Headache Classification Committee of the International Headache Society (IHS). The International Classification of Headache Disorders, 3rd edition (beta version). Cephalalgia 2013; 33: 629-808.

2. de Tommaso M, Valeriani M, Guido M, et al. Abnormal brain processing of cutaneous pain in patients with chronic migraine. Pain 2003; 101: 25-32.

3. Coppola $\mathrm{G}$ and Schoenen J. Cortical excitability in chronic migraine. Curr Pain Headache Rep 2012; 16: 93-100.

4. Price DD. Psychological and neural mechanisms of the affective dimension of pain. Science 2000; 288: 1769-1772.

5. Legrain V, Iannetti GD, Plaghki L, et al. The pain matrix reloaded: A salience detection system for the body. Prog Neurobiol 2011; 93: 111-124.

6. Xue T, Yuan K, Zhao L, et al. Intrinsic brain network abnormalities in migraines without aura revealed in resting-state fMRI. PLoS One 2012; 7: e52927.

7. Hubbard CS, Khan SA, Keaser ML, et al. Altered brain structure and function correlate with disease severity and pain catastrophizing in migraine patients. eNeuro 2014; 1: e20.14.
8. Schwedt TJ, Chiang CC, Chong CD, et al. Functional MRI of migraine. Lancet Neurol 2015; 14: 81-91.

9. Fumal A, Laureys S, Di Clemente L, et al. Orbitofrontal cortex involvement in chronic analgesic-overuse headache evolving from episodic migraine. Brain 2006; 129: 543-550.

10. Grazzi L, Chiapparini L, Ferraro S, et al. Chronic migraine with medication overuse pre-post withdrawal of symptomatic medication: Clinical results and FMRI correlations. Headache 2010; 50: 998-1004.

11. Lai TH, Chou KH, Fuh JL, et al. Gray matter changes related to medication overuse in patients with chronic migraine. Cephalalgia 2016; 36: 1324-1333.

12. Riederer F, Schaer M, Gantenbein AR, et al. Cortical alterations in medication-overuse headache. Headache 2017; 57: 255-265.

13. Chanraud S, Di Scala G, Dilharreguy B, et al. Brain functional connectivity and morphology changes in medication-overuse headache: Clue for dependence-related processes? Cephalalgia 2014; 34: 605-615.

14. Bogdanov VB, Vigano A, Noirhomme Q, et al. Cerebral responses and role of the prefrontal cortex in conditioned pain modulation: An fMRI study in healthy subjects. Behav Brain Res 2015; 281: 187-198.

15. Deichmann R, Schwarzbauer C and Turner R. Optimisation of the 3D MDEFT sequence for anatomical brain imaging: Technical implications at 1.5 and $3 \mathrm{~T}$. Neuroimage 2004; 21: 757-767.

16. Andersson JL, Hutton C, Ashburner J, et al. Modeling geometric deformations in EPI time series. Neuroimage 2001; 13: 903-919.

17. Hutton C, Bork A, Josephs $\mathrm{O}$, et al. Image distortion correction in fMRI: A quantitative evaluation. Neuroimage 2002; 16: 217-240.

18. Ashburner $\mathbf{J}$ and Friston KJ. Unified segmentation. Neuroimage 2005; 26: 839-851.

19. Downar J, Mikulis DJ and Davis KD. Neural correlates of the prolonged salience of painful stimulation. Neuroimage 2003; 20: 1540-1551.

20. Micieli G, Tassorelli C, Bosone D, et al. Increased cerebral blood flow velocity induced by cold pressor test in migraine: A possible basis for pathogenesis? Cephalalgia 1995; 15: 494-498.

21. Amin FM, Hougaard A, Magon S, et al. Altered thalamic connectivity during spontaneous attacks of migraine without aura: A resting-state fMRI study. Cephalalgia 2018; 38: 1237-1244. 
22. Ge HT, Liu HX, Xiang J, et al. Abnormal cortical activation in females with acute migraine: A magnetoencephalography study. Clin Neurophysiol 2015; 126: 170-179.

23. Budell L, Kunz M, Jackson PL, et al. Mirroring pain in the brain: Emotional expression versus motor imitation. PLoS One 2015; 10(2): e0107526.

24. Schwedt TJ. Multisensory integration in migraine. Curr Opin Neurol 2013; 26: 248-253.

25. Louter MA, Pijpers JA, Wardenaar KJ, et al. Symptom dimensions of affective disorders in migraine patients. $J$ Psychosom Res 2015; 79: 458-463.

26. Tedeschi G, Russo A, Conte F, et al. Increased interictal visual network connectivity in patients with migraine with aura. Cephalalgia 2016; 36: 139-147.

27. Boulloche N, Denuelle M, Payoux P, et al. Photophobia in migraine: An interictal PET study of cortical hyperexcitability and its modulation by pain. J Neurol Neurosurg Psychiatry 2010; 81: 978-984.

28. Schwedt TJ, Chong CD, Chiang CC, et al. Enhanced pain-induced activity of pain-processing regions in a case-control study of episodic migraine. Cephalalgia 2014; 34: 947-958.

29. Goldberg, II, Harel M and Malach R. When the brain loses its self: Prefrontal inactivation during sensorimotor processing. Neuron 2006; 50: 329-339.

30. Cruccu G, Garcia-Larrea L, Hansson P, et al. EAN guidelines on central neurostimulation therapy in chronic pain conditions. Eur J Neurol 2016; 23: 1489-1499.

31. Riederer F, Gantenbein AR, Marti M, et al. Decrease of gray matter volume in the midbrain is associated with treatment response in medication-overuse headache: Possible influence of orbitofrontal cortex. J Neurosci 2013; 33: 15343-15349.

32. Coppola G, Curra A, Di Lorenzo C, et al. Abnormal cortical responses to somatosensory stimulation in medication-overuse headache. BMC Neurol 2010; 10: 1471-2377.

33. Ayzenberg I, Obermann M, Nyhuis P, et al. Central sensitization of the trigeminal and somatic nociceptive systems in medication overuse headache mainly involves cerebral supraspinal structures. Cephalalgia 2006; 26: 1106-1114.

34. de Tommaso M, Ambrosini A, Brighina F, et al. Altered processing of sensory stimuli in patients with migraine. Nat Rev Neurol 2014; 10: 144-155.

35. Riederer F, Marti M, Luechinger R, et al. Grey matter changes associated with medication-overuse headache: Correlations with disease related disability and anxiety. World J Biol Psychiatry 2012; 13: 517-525.

36. Chen Z, Jia Z, Chen X, et al. Volumetric abnormalities of thalamic subnuclei in medication-overuse headache. J Headache Pain 2017; 18: 82.

37. Schoenen J. Is chronic migraine a never-ending migraine attack? Pain 2010; 152: 239-240.

38. Chen WT, Wang SJ, Fuh JL, et al. Visual cortex excitability and plasticity associated with remission from chronic to episodic migraine. Cephalalgia 2012; 32: 537-543.

39. Chen B, He Y, Xia L, et al. Cortical plasticity between the pain and pain-free phases in patients with episodic tension-type headache. J Headache Pain 2016; 17: 105. 